\title{
Electrospray Ionization Mass Spectrometric Observation of Oligomers in Paal-Knorr Synthesis of 2,5-Dimethyl-1-phenylpyrrole
}

\author{
So-Young Park, Man-Seog Chun, Jin-Su Song, and Hie-Joon Kim* \\ Department of Chemistry, Seoul National University, Gwanak-gu, Seoul 151-747, Korea. *E-mail: hjkim1@snu.ac.kr \\ ${ }^{\dagger}$ Busan Science Academy, Danggam 3-dong, Busanjin-gu, Busan 614-822, Korea \\ Received November 15, 2004
}

\begin{abstract}
Electrospray ionization mass spectrometry (ESI MS) was used, along with gas chromatography-mass spectrometry (GC-MS), to monitor Paal-Knorr synthesis of 2,5-dimethyl-1-phenylpyrrole by condensation of aniline with 2,5-hexanedione. In addition to 2,5-dimethyl-1-phenylpyrrole observed as a single spot by TLC, unexpected dimer size compounds were observed by GC-MS. Dimers and trimers were observed by ESI MS. ESI tandem mass spectrometry was used to select plausible structures for the dimer. ESI MS with or without liquid chromatographic separation is useful for observing oligomeric byproducts with low volatility produced in organic reactions.
\end{abstract}

Key Words : ESI MS, GC-MS, Paal-Knorr synthesis, 2,5-Dimethyl-1-phenylpyrrole, Oligomer

\section{Introduction}

Thin layer chromatography (TLC) has been widely used, due to its simplicity and low cost, to monitor reaction products in organic synthesis. Progress of a reaction can be better monitored by high performance liquid chromatography (HPLC) particularly using photodiode array detection in the UV-visible range. However, information about the identity of the products is limited in both TLC and HPLC unless chromatographic retention time and the absorption spectrum of authentic compounds are known.

Mass spectrometry (MS) offers important mass information not available in spectrophotometric methods. Mass selective detection (MSD) can be used, either in gas chromatography (GC) or in liquid chromatography (LC), for highly specific monitoring of organic compounds including drugs. ${ }^{1}$ Mass data can also be used in investigating polymerization mechanism. ${ }^{2,3}$ GC-MS with electron impact (EI) ionization could be used to observe low molecular weight byproducts including possible oligomers of the primary reaction products. ${ }^{4}$ Higher molecular weight oligomers with low volatility could be observed by LC-MS.$^{5,6}$ As a matter of fact, with the ability of electrospray ionization mass spectrometry (ESI MS) to produce analyte ions from liquid samples and the high resolution of modern mass analyzers, mass profile of a complex reaction mixture can be obtained without LC separation.

In this paper, using simple Paal-Knorr synthesis of 2,5dimethyl-1-phenylpyrrole (MW 171.10 Da) from aniline and 2,5-hexanedione (Fig. 1), we explore the capabilities of ESI MS techniques for monitoring organic reactions. Paal-Knorr synthesis is one of several cyclization reactions forming nitrogen heterocycles and is used to make pyrroles by heating 1,4-dicarbonyl compounds with ammonia or primary amine. Wolthuis et al. synthesized 2,5-dimethyl-1phenylpyrrole by Paal-Knorr reaction in 1965 with $72 \%$

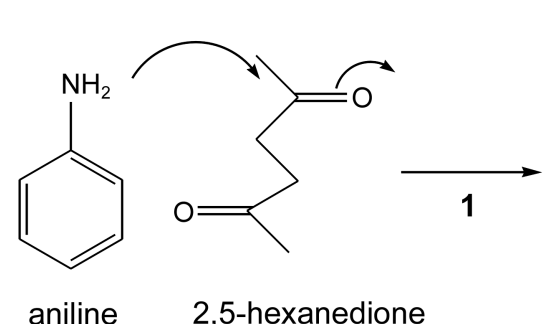<smiles>CC(=O)NC(C)(O)CCC(C)(C)O</smiles>

aniline 2,5-hexanedione 2
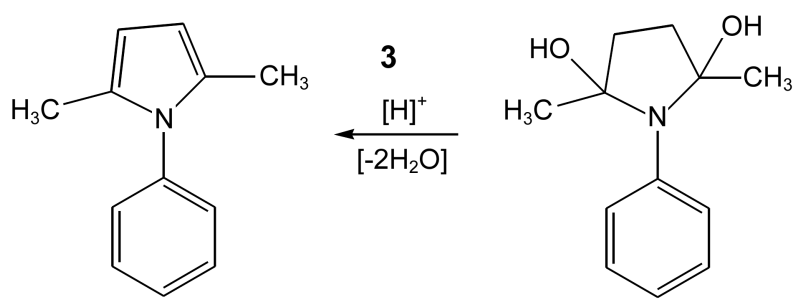

2,5-dimethyl-1-phenylpyrrole

Figure 1. Synthetic mechanism of 2,5-dimethyl-phenylpyrrole from aniline and 2,5-hexanedione.

yield. ${ }^{7}$ They confirmed purity of the recrystallized product using nuclear magnetic resonance (NMR) and infrared (IR) spectroscopy as well as TLC and melting point measurement. Shaw et al. presented a simple reaction scheme for this reaction (Fig. 1) and reported a $52 \%$ yield. $^{8}$

We noted that the single spot on the TLC plate for 2,5dimethyl-1-phenylpyrrole, which was initially observable only by UV, over time became colored and observable with the naked eye. Clearly, further chemical changes, not reported in the literature, were taking place. We report mass spectrometric evidence for formation of dimers and trimers not available by TLC or HPLC observations. 


\section{Experimental Section}

Chemicals. Aniline (99.5+\%, A.C.S. reagent), acetonylacetone (98+\%), and dithranol were from Aldrich Chemical Co. (Milwaukee, WI, USA). Hydrochloric acid (extra pure reagent) and anhydrous ethyl alcohol (99.9\%) were from Dajung Chemicals \& Metals Co. Ltd. (Daegu, Korea). HPLC grade methanol was from Fisher Scientific Korea Ltd. (Seoul, Korea). Distilled water was prepared using Millipore Milli-Q system (Billercia, MA, USA).

Reaction. $1.86 \mathrm{~mL}$ aniline $(20 \mathrm{mmol})$ was added to a 100 $\mathrm{mL}$ round bottom flask equipped with a reflux condenser. $2.37 \mathrm{~mL}$ 2,5-hexanedione (20 mmol), $5 \mathrm{~mL}$ methanol, and 6 drops of concentrated $\mathrm{HCl}$ were added, and the mixture was stirred with a magnetic stirrer while heating using a heating mantle $\left(80^{\circ} \mathrm{C}\right)$. After $5 \mathrm{~min}$ reflux, $0.5 \mathrm{~mL}$ aliquot was withdrawn with a pasture pipette. After another $10 \mathrm{~min}$, the reaction mixture was transferred to a $100 \mathrm{~mL}$ beaker containing $50 \mathrm{~mL}$ of cold $0.5 \mathrm{M} \mathrm{HCl}$. After several min cooling in ice bath, crystals formed were collected with a Hirsch funnel under vacuum and crystallized using methanol/distilled water $(9: 1, \mathrm{v} / \mathrm{v})$.

$0.5 \mathrm{~mL}$ methanol was added to the $0.5 \mathrm{~mL}$ aliquot of the 5 min reflux sample, and the mixture was further diluted 100fold with methanol (hereafter referred to as 5 min sample). $10 \mathrm{mg}$ crystallized product was dissolved in $1 \mathrm{~mL}$ methanol, and the solution was diluted 100-fold with methanol (referred to as crystallized sample). Both samples were analyzed by GC-MS and ESI MS.

TLC. Silica gel plate (silicagel $60 \mathrm{~F}_{254}$, Merck, Rahway, NJ, USA) and ethanol/hexane $(1: 3, \mathrm{v} / \mathrm{v})$ were used. UV lamp (Spectroline ENF-240C, Spectronics Corp., Westbury, NY, USA) was used to visualize the spot.

GC-MS. $5 \mu \mathrm{L}$ of both samples were injected into the gas chromatograph (Agilent Technologies 6890N Network GC system) equipped with 5973 Network Mass Selective Detector operating at $70 \mathrm{eV}$ electron impact mode. The 30.0 $\mathrm{m} \times 250 \mu \mathrm{m} \times 0.25 \mu \mathrm{m}$ capillary was HP $19091 \mathrm{~S}-433$ coated with HP-5MS 5\% phenyl methyl siloxane. Flow rate of He was $1.0 \mathrm{~mL} \mathrm{~min} \mathrm{~m}^{-1}$ at $13.3 \mathrm{psi}$. Temperature of the column was varied from 75 to $300{ }^{\circ} \mathrm{C}$. The mass scan range was $\mathrm{m} / \mathrm{z}$ 40-400.

ESI MS. ESI MS experiments were performed at Busan Science Academy using an instrument equipped with 1525 Binary HPLC pump and a Waters ZQ single quadrupole mass spectrometer with electrospray ionization source (Waters, Manchester, UK). The ionization source block temperature was kept at $70{ }^{\circ} \mathrm{C}$ and desolvation temperature was $200{ }^{\circ} \mathrm{C}$. Flow rate of the desolvation gas and cone gas was $150 \mathrm{~L} / \mathrm{h}$ and $50 \mathrm{~L} / \mathrm{h}$, respectively. Following voltages were used for ESI MS in the positive mode: capillary voltage, 3.0-3.08 kV (adjustable); cone voltage, 20-30 V; extractor, $5 \mathrm{~V}$; RF lens, $0.5 \mathrm{~V}$. When LC separation was not necessary, $10 \mu \mathrm{L}$ sample was injected using a Rheodyne injector while methanol-water $(80: 20$, v/v) was continuously introduced into the ESI MS system at $0.1 \mathrm{~mL} / \mathrm{min}$ flow rate. When separation was needed, a reversed-phase column (Xterra MS C18, $3.5 \mu \mathrm{m}, 2.1 \times 150 \mathrm{~mm}$ ) was used with $90 \%$ methanol as eluent. Flow rate was $0.1 \mathrm{~mL} / \mathrm{min}$.

ESI MS/MS. ESI tandem mass spectrometry was performed using a triple quadrupole mass spectrometer (Quattro-LC, Micromass, Manchester, UK) at the Seoul National University Center for Inter-University Research Facilities. Argon was the collision gas and collision voltage was adjusted between 10 and $40 \mathrm{~V}$. Sample flow rate was $10 \mu \mathrm{L} /$ min. Block temperature of the ionization source was $70^{\circ} \mathrm{C}$. Capillary voltage and cone voltage were $3.7 \mathrm{kV}$ and $30 \mathrm{~V}$, respectively.

\section{Results and Discussion}

The present work was prompted by observation of color change in the TLC spot with Rf value of 0.85 for 2,5dimethyl-1-phenylpyrrole synthesized by the Paal-Knorr reaction. The spot, which was initially observable only by UV, became colored reddish brown. Further chemical changes were obvious; however, oligomerization was not suspected. Further reactions taking place from 2,5-dimethyl1-phenylpyrrole were monitored using the reaction mixture before separation by crystallization or TLC.

GC-MS. Figure 2a shows a total ion chromatogram (TIC) obtained by GC-MS from $5 \mu \mathrm{L}$ injection of the $5 \mathrm{~min}$ sample. The EI spectrum for peak A showed that it is from aniline. The strongest peak (B), was observed at $11.4 \mathrm{~min}$ and smaller peaks were observed at 22.5 (C) and $23.9 \mathrm{~min}$ (D). No further peaks were observed after $25 \mathrm{~min}$. The EI fragmentation pattern for peak B (Fig. 2b) was identical to that from 2,5-dimethyl-1-phenylpyrrole in the mass spectral library. Figure $2 \mathrm{~b}$ shows signals at $\mathrm{m} / \mathrm{z} 170$ for $[\mathrm{M}-\mathrm{H}]^{+}$and 171 for $\mathrm{M}^{+\mathrm{h}}$ as well as their fragments. The $\mathrm{m} / \mathrm{z} 77$ peak is for $\mathrm{C}_{6} \mathrm{H}_{5}{ }^{+}$derived from cleavage of the benzene ring. The relative intensity of aniline and the major product showed that the synthetic reaction was almost complete after $5 \mathrm{~min}$. Therefore, subsequent analyses were performed using the 5 min sample.

Figures $2 \mathrm{c}$ and $2 \mathrm{~d}$ show EI spectra for peaks C and D. Their parent molecular weights, 342 and $340 \mathrm{Da}$, suggest that they are dimer size compounds related to 2,5-dimethyl1-phenylpyrrole. Their EI fragmentation patterns were quite different from each other. For example, the $\mathrm{m} / \mathrm{z} 183$ ion was dominant in peak D, but not present in C. EI mass spectra for the dimer-size compounds were not available in the mass spectral library.

Figures 2a shows that the 342 Da dimer is about 4 times more abundant than the $340 \mathrm{Da}$ dimer. The difference in the

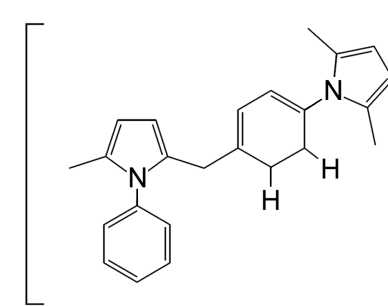

(dimer I; $342 \mathrm{Da}$ )
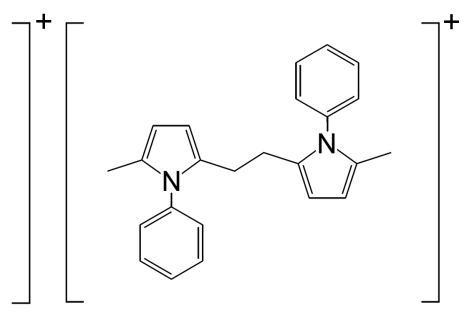

(dimer II; $340 \mathrm{Da})$ 

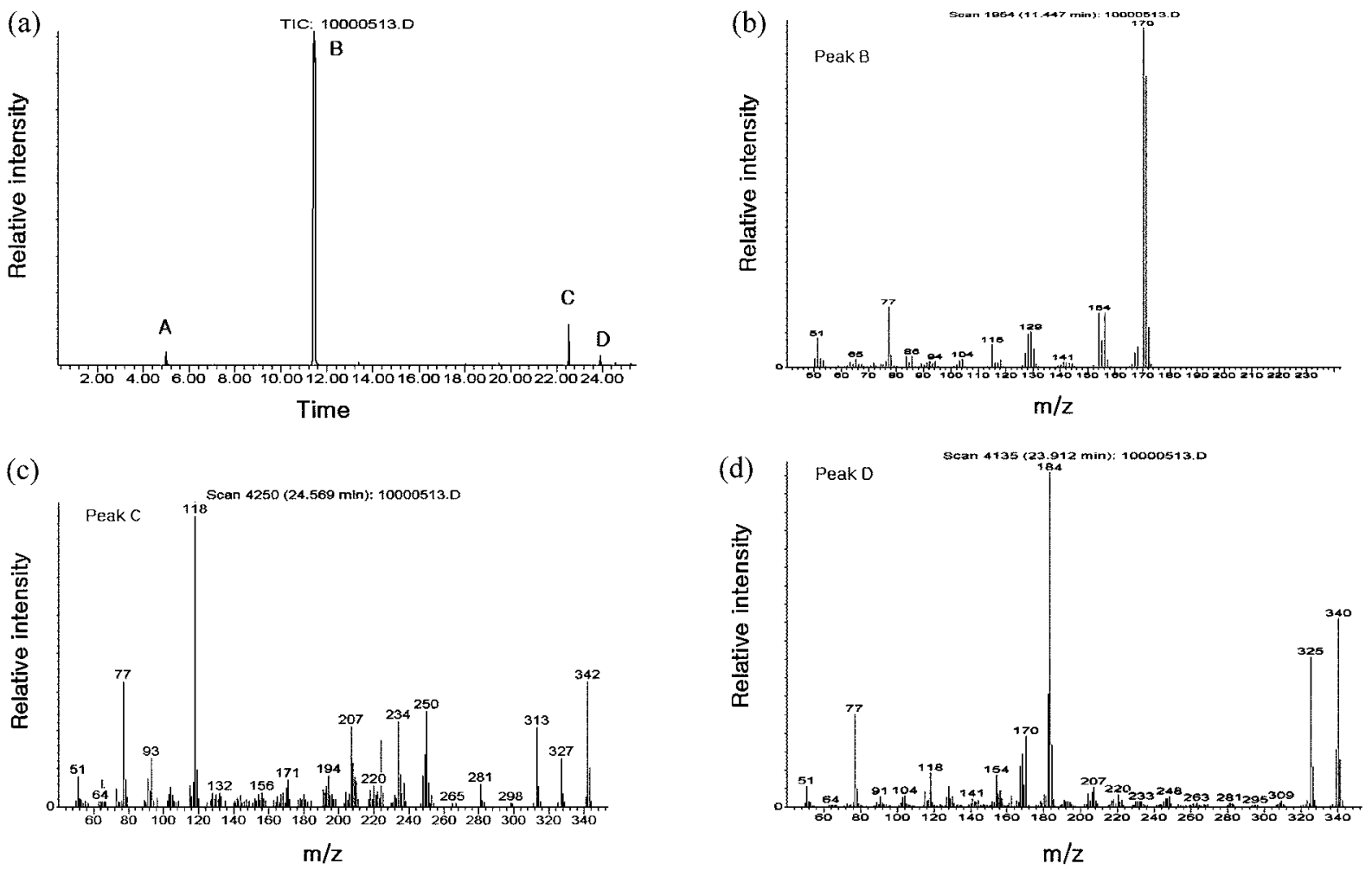

Figure 2. Total ion chromatogram and EI spectra obtained by GC-MS from the 5 min reaction mixture.

EI pattern suggested that dimerization took place through multiple pathways. Candidate structures for 342 and $340 \mathrm{Da}$ dimers are shown below:

Presumably formation of dimer I involves fusion of the pyrrole and the phenyl parts as well as hydrogenation of one of the benzene rings. Such hydrogenation has been reported in gamma-irradiation induced cross-linking of aromatic amino acids. ${ }^{9}$ The $\mathrm{m} / \mathrm{z} 327$ and 313 ions probably involve removal of one and two methyl groups from the pyrrole ring. The $\mathrm{m} / \mathrm{z} 250$ ion is consistent with removal of the pyrrole. The $\mathrm{m} / \mathrm{z} 118$ ion could not be accounted for. It might involve rupture of the benzene ring.

Dimer II is probably made by fusion of the pyrrole parts through hydrogen abstraction from the methyl group. The proposed structure for dimer II with two adjacent methylene groups is consistent with fragment ions observed at $\mathrm{m} / \mathrm{z} 170$ and 184 . The $\mathrm{m} / \mathrm{z} 325$ ion is due to removal of a methyl group.

The presence of trimeric or tetrameric compounds could not be tested, because they probably have low volatility and are not amenable to GC-MS even if they were produced. Anyhow, the GC-MS results clearly indicated that<smiles>CCc1ccc(C)n1-c1ccccc1</smiles>

dimerization of 2,5-dimethyl-1-phenylpyrrole took place. Furthermore, the EI spectra provided insight as to the possible dimerization pathways.

ESI MS. Figure 3a shows ESI mass spectrum obtained in the positive ion mode by injecting $10 \mu \mathrm{L}$ crystallized sample using a Rheodyne injector while $80 \%$ methanol was continuously introduced into the ESI MS system. The major peak at $\mathrm{m} / \mathrm{z} 172.2$ is from $[\mathrm{M}+\mathrm{H}]^{+}$ion of 2,5-dimethyl-1-phenylpyrrole.

The spectrum also shows a small peak at $\mathrm{m} / \mathrm{z} 170.1$. To see whether the 170.1 ion is derived from the 172.2 ion during the mass spectrometric analysis or represents a compound distinct from the 172.2 peak, the crystallized sample was separated by LC prior to ESI MS detection. Mass selective detection at $\mathrm{m} / \mathrm{z} 170.1$ and 172.2 produced two separate chromatograms showing $\mathrm{m} / \mathrm{z} 170.1$ eluting at $7.5 \mathrm{~min}$ and 172.2 at $10.3 \mathrm{~min}$ (data not shown). Clearly, the $\mathrm{m} / \mathrm{z} 170.1$ peak in Figure $3 \mathrm{a}$ is not $[\mathrm{M}-\mathrm{H}]^{+}$generated from 2,5dimethyl-1-phenylpyrrole during the ionization process. A possible reaction scheme for conversion of 2,5-dimethyl-1phenylpyrrole to 2,5-dimethylene-1-phenyl-2,5-dihydro-1Hpyrrole, a candidate compound with 169.09 Da MW $\left([\mathrm{M}+\mathrm{H}]^{+}\right.$observed at 170.19), is shown in Figure 4.

Figure $3 \mathrm{~b}$ shows ESI mass spectrum obtained similarly from the $5 \mathrm{~min}$ sample. Peaks at $\mathrm{m} / \mathrm{z} 170.2$ and 172.1 were observed as in Figure 3a. Additionally, several strong peaks were observed at $\mathrm{m} / \mathrm{z} 250.3,279.2,343.2$, and 522.2. The 343 peak corresponds to $[\mathrm{M}+\mathrm{H}]^{+}$of the dimer peak observed at 342 by GC-MS (Fig. 2c). No significant signal was obtained at $\mathrm{m} / \mathrm{z} 341$ for the $340 \mathrm{Da}$ dimer observed by GCMS. The $\mathrm{m} / \mathrm{z} 250.3$ peak was also observed in the EI 

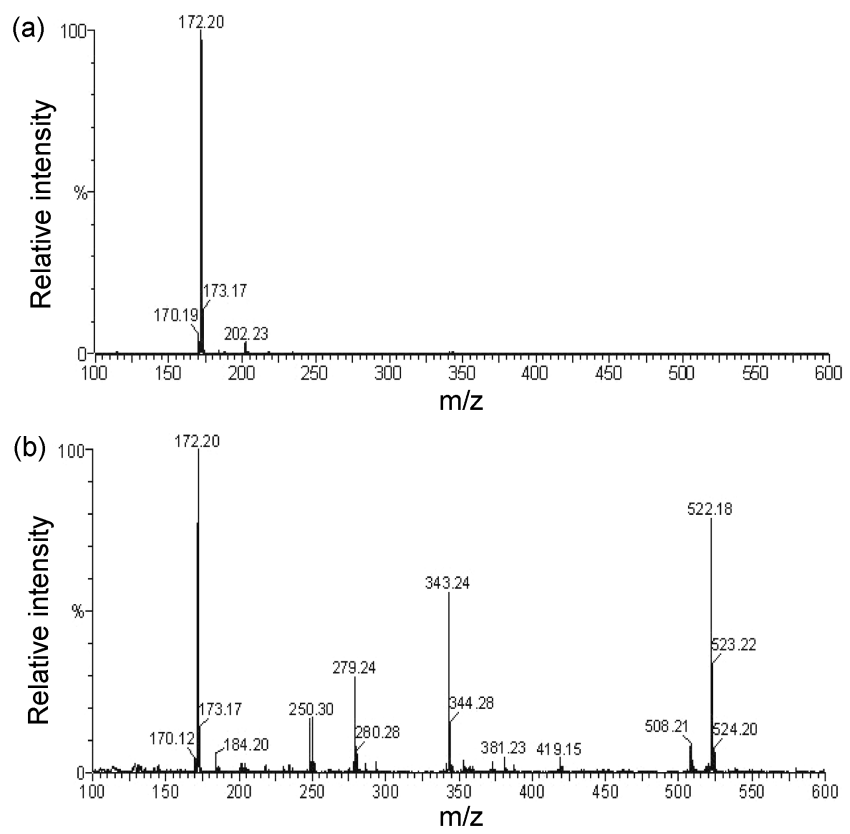

Figure 3. ESI mass spectra from the crystallized product (a) and the 5 min reaction mixture (b).

spectrum (Fig. 2c). The m/z 279.2 peak could not be accounted for. The 522 and neighboring peaks appear to be trimer size oligomers.

To make sure that the oligomeric peaks were not due to cluster ions produced during the ESI process, ESI mass spectra were obtained from the 5 min sample after LC separation. The MSD results showed that the $\mathrm{m} / \mathrm{z} 172,343$ and 522 peaks were eluted at 7.0, 15.3 and $18.6 \mathrm{~min}$, respectively (data not shown), suggesting that they represent separate compound. If it were clusters, they should have been observed in the ESI spectrum from the recrystallized monomer (Fig. 3a). Detection of dimer size compounds by ESI MS is consistent with the GC-MS result. The trimer was not observed by GC-MS probably due to its low volatility.

ESI MS/MS. The full scan mass spectrum from the $5 \mathrm{~min}$ sample showed, in addition to the predominant $\mathrm{m} / \mathrm{z} 172$ ion,

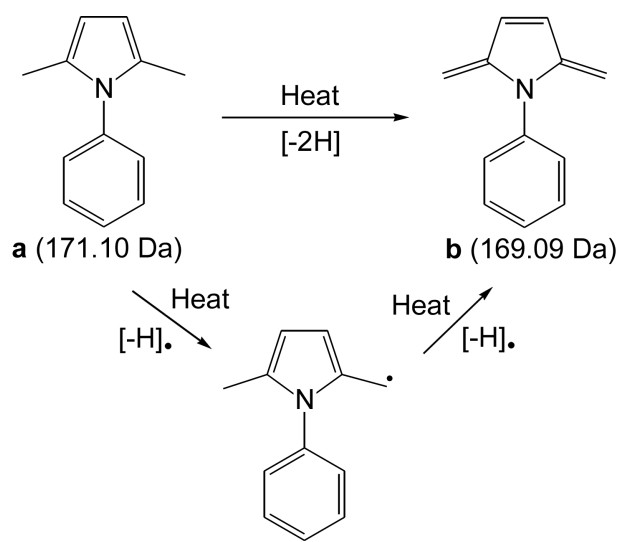

Figure 4. Conversion of 2,5-dimethyl-1-phenylpyrrole to 2,5dimethylene phenyl-1H-pyrrole.

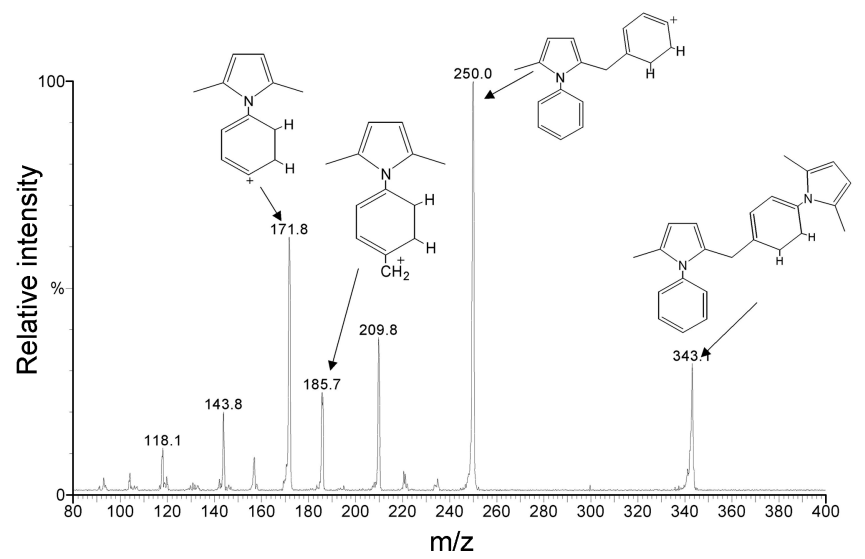

Figure 5. ESI tandem mass spectrum derived from the $\mathrm{m} / \mathrm{z} 343$ ion.

signals at $170,341,343,522$, and 689. Figure 5 shows ESI tandem mass spectrum obtained from fragmentation of the $\mathrm{m} / \mathrm{z} 343$ ion (dimer I). Suggested structures for the 172, 186, and 250 fragments are consistent with the structure for dimer I above. The $\mathrm{m} / \mathrm{z} 210$ ion probably involves rupture of the benzene ring. These fragment ions were also observed by GC-MS (Fig. 2c). The m/z 343 ion was also observed from purified 2,5-dimethyl-1-phenylpyrrole when left in solution. Thus dimer I might be responsible for the color change in the TLC spot.

The tandem mass spectrum from $\mathrm{m} / \mathrm{z} 341$ ion (dimer II) showed daughter ions at $\mathrm{m} / \mathrm{z} 170$ and 184, which were also observed by GC-MS. The tandem mass spectrum from the trimer size $\mathrm{m} / \mathrm{z} 522$ ion showed daughter ions at $\mathrm{m} / \mathrm{z} 118$ and 184 , which were observed by GC-MS as predominant ions in Fig. 2c and 2d. Observation of these common fragments suggests that the $\mathrm{m} / \mathrm{z} 522$ ion is a trimer derived from 2,5dimethyl-1-phenylpyrrole.

In conclusion, combined mass spectrometric results show that 2,5-dimethyl-1-phenylpyrrole is rapidly produced and undergoes oligomerization reactions. ESI MS is useful for monitoring high molecular weight oligomers produced in organic reactions.

Acknowledgment. S.-Y. Park and J.-S. Song acknowledge the financial support from the Brain Korea 21 fellowship.

\section{References}

1. Yoon, K.-H.; Kim, W.; Park, J.; Kim, H.-J. Bull. Korean Chem. Soc. 2004, 25, 878-880.

2. Kim, H.-J.; Lee, J.-K.; Park, S. J.; Ro, H. W.; Yoo, D. Y.; Yoon, D. Y. Anal. Chem. 2000, 72, 5673-5678.

3. Kim, H.-J.; Lee, J.-K.; Kim, J.-B.; Park, E. S.; Park, S. J.; Yoo, D. Y.; Yoon, D. Y. J. Am. Chem. Soc. 2001, 123, 12121-12122.

4. Pasch, H.; Gores, F. Polymer 1995, 36, 1999-2005.

5. Wei, X.; Zongming, G. Chem. Mater. 2001, 13, 2979-2990.

6. Koc, H.; Mei-Heng, M. Anal. Chem. 2002, 74, 4734-4740.

7. Wolthuis, E.; Jagt, D. V.; Mels, S.; De Boer, A. J. Org. Chem. 1965, 30, 190-193.

8. Shaw, D. J.; Wood, W. F. J. Chem. Ed. 1992, 69, A313.

9. Kim, H.-J.; Mee, L. K.; Adelstein, S. J.; Taub, I. A.; Carr, S. A.; Reinhold, V. N. Radiat. Res. 1984, 100, 30-40. 